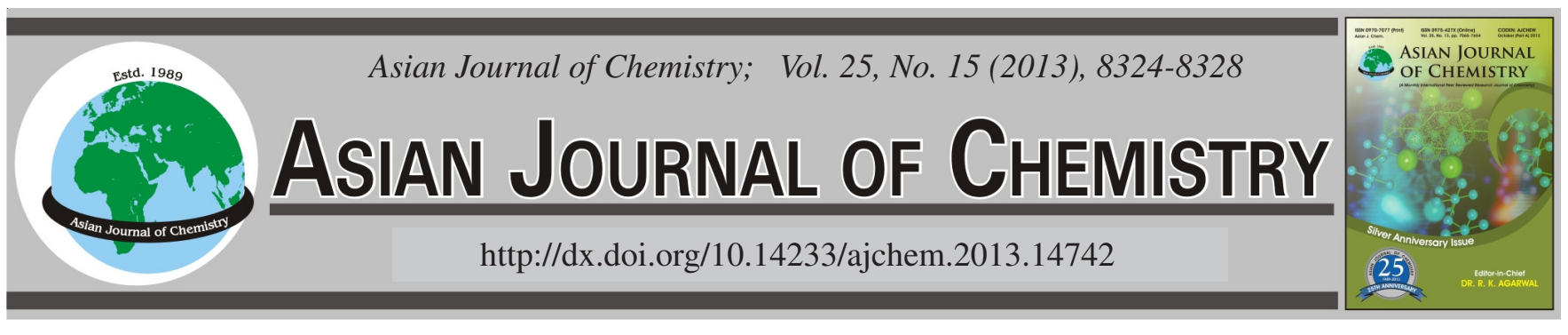

\title{
Utilization of Zeolite Imidazolate Framework as an Adsorbent for the Removal of Dye from Aqueous Solution
}

\author{
XIAO-Zhen KAng ${ }^{1}$, Zheng-Wei Song ${ }^{2}$, Qi ShI ${ }^{1, *}$ and Jin-XIANG Dong ${ }^{1, *}$
}

${ }^{1}$ Research Institute of Special Chemicals, Taiyuan University of Technology, Taiyuan 030024, Shanxi Province, P.R. China ${ }^{2}$ Department of Coal Chemical, Yili Institute of Technician Training, Yili 835000, Xinjiang Province, P.R. China

*Corresponding authors: Tel./Fax: +86 351 611178; E-mail: shiqi594@163.com; dongjinxiangwork@ hotmail.com

\begin{abstract}
In this work, we have described the adsorption of dye acid blue 40 and methylene blue from aqueous solution onto isotypic zeolite imidazolate frameworks (ZIFs) such as ZIF-8 $\left[\mathrm{Zn}(\mathrm{mim})_{2}\right]$ and ZIF-67 $\left[\mathrm{Co}(\mathrm{mim})_{2}\right]$. The results suggested that the charged sites at the external surface of zeolite imidazolate frameworks play a key role in dye adsorption. A similar adsorption capacity is observed for the uptake cationic dye methylene blue between ZIF-8 and ZIF-67, involving electrostatic interactions between the same N-extremities (belonging to mim linkers) and positively charged methylene blue. For anionic dye acid blue 40, the adsorption capacity for ZIF-67 is nearly three times higher than that of ZIF-8, involving electrostatic interactions between low-coordinated metal cations of zeolite imidazolate frameworks and negatively charged groups of acid blue 40 and Co cations in ZIF-67 have higher affinity with the $\mathrm{SO}_{3}^{-}$groups of acid blue 40 .
\end{abstract}

Key Words: Adsorbent, Dye, Liquid phase adsorption, Metal-organic frameworks, External surface.

\section{INTRODUCTION}

Zeolitic imidazolate frameworks (ZIFs) are a new subclass of porous metal-organic frameworks (MOFs) which frequently have expanded zeolite topologies ${ }^{1-6}$. By combining and enhancing the desirable features of both zeolites and metalorganic frameworks, ZIFs are considered to be versatile materials for gas storage ${ }^{7}$, separations $\mathrm{s}^{8,9}$ and catalysis ${ }^{10}$, as well as having other potential applications ${ }^{11}$. When compared with many other metal-organic frameworks, ZIFs show exceptional stability and resistance to boiling organic solvents and water, due to their intrinsically hydrophobic nature and strong bonds ${ }^{3}$. They have also been demonstrated to be more stable in boiling aqueous solution of $\mathrm{NaOH}$ than many low-silica zeolites ${ }^{3}$. In fact, the bulk of investigations on ZIFs have involved their application in gas adsorption and separation ${ }^{4,7-9}$. Due to their above-mentioned stability, they hold great promise in liquid phase adsorption and separation and may help to solve pressing environmental problems.

So far, there are a limited number of reports on ZIFs as adsorbents for liquid phase adsorption and separation ${ }^{12-14}$. For example, butanol separation from aqueous mixture with ZIF-8 was reported by Denayer and co-workers and the large internal porosity of ZIF-8 is utilized ${ }^{13}$. Chizallet et al. ${ }^{15}$ demonstrated that a great variety of sites are located on the external surface of ZIFs or at defects, such as low-coordinated metal cations, as well as free $\mathrm{N}$ - extremities belonging to imidazolate linkers. Thus, it was deemed of interest to ascertain whether these sites of ZIFs at the external surface might play a role in the liquid phase adsorption and separation.

Shortages of water are a common problem worldwide that is exacerbated by the generation of large amounts of wastewater containing a variety of pollutants. Synthetic dyes are an important group of pollutants that are generated from many industries including textile, leather, paper, printing, dyestuff, plastic, food and cosmetics ${ }^{16}$.

In this work, we report the results of the adsorption of dye from aqueous solution by using ZIFs. Dyes Acid Blue 40 (AB40, anionic dye) and methylene blue (MB, cationic dye) were chosen as adsorbate and their structures are shown in Scheme-I. The choice is motivated by their charge (AB40 is negative, MB positive) after dyes are dissolved in water solution, which is helpful for a systematic study of the effect of external surface sites with ZIFs as adsorbents in the liquid phase adsorption and separation. In fact, the wastewater contained an anthraquinone dyestuff-AB40 that are very often used in textile industry ${ }^{17,18}$. Methylene blue is also the most commonly used substance for dying cotton, wood and silk ${ }^{19}$. Several adsorbents, including activated carbon and titania have been studied for the removal of both $\mathrm{AB} 40^{20,21}$ and MB dyes ${ }^{19,21}$.

The adsorbents tested were ZIF-8 (also known as MAF$4^{2}$ ) and ZIF-67 ${ }^{3}$. ZIF-8[Zn $(\operatorname{mim})_{2}$, mim = 2-methylimidazolate $]$ 


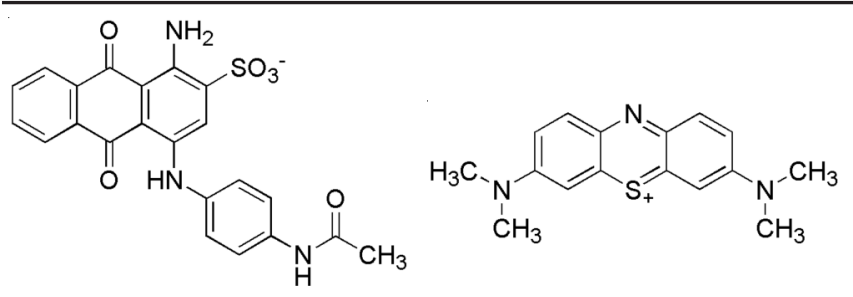

Scheme-I: Structures of the acid blue 40 (left) and methylene blue (right)

and ZIF-67 [Co(mim) $\left.)_{2}\right]$ are representative of ZIFs materials with a sod (zeolite sodalite) topology, which is formed by fourring and six-ring $\mathrm{ZnN}_{4}$ or $\mathrm{CoN}_{4}$ clusters linked by 2-methyl imidazolate ${ }^{3}$. The effect of metal ions can be understood on the adsorption of dyes over isotypic ZIF-8 (Zn) and ZIF-67 (Co).

\section{EXPERIMENTAL}

2-Methylimidazole $\left(\mathrm{C}_{4} \mathrm{H}_{6} \mathrm{~N}_{2}, 99.0 \%\right)$, zinc acetate dihydrate $(98.0 \%)$ were purchased from Sigma-Aldrich Chemical Co. and cobalt acetate tetrahydrate ( $99.5 \%$ ), methanol $\left(\mathrm{CH}_{3} \mathrm{OH}, 99.5 \%\right)$ were obtained from Sinopharm Group Chemical Reagent Co. acid blue $40\left(\mathrm{AB} 40, \mathrm{C}_{22} \mathrm{H}_{17} \mathrm{~N}_{3} \mathrm{O}_{6} \mathrm{SNa}\right.$, CI 62125) was obtained from Sigma-Aldrich Chemical Co. and methylene blue (MB, $\mathrm{C}_{16} \mathrm{H}_{18} \mathrm{ClN}_{3} \mathrm{~S}$, CI 52015) was obtained from $\mathrm{ABCR} \mathrm{GmbH} \& \mathrm{CO}$. KG. These dyes were used as received without further purification. PXRD patterns were recorded with a X-ray diffractometer (Rigaku, MiniFlexII) using $\mathrm{CuK}_{\alpha}$ radiation $(\lambda=1.5418 \AA$ ). The dye concentrations of the solutions were measured by UV-visible spectrophotometer (Shanghai Precision \& Scientific, UV765).

Synthesis: $\mathrm{ZIF}-8, \mathrm{Zn}(\mathrm{OAc})_{2} \cdot 2 \mathrm{H}_{2} \mathrm{O}(3.52 \mathrm{~g}, 16 \mathrm{mmol})$ and 2-methylimidazole (3.94 g, $48 \mathrm{mmol})$ were dissolved in methanol $(120 \mathrm{~mL})$ in a $150 \mathrm{~mL}$ Teflon-lined stainless steel autoclave at $120^{\circ} \mathrm{C}$ for $72 \mathrm{~h}$. After cooling to room temperature, the solid products were separated by filtration and washed with ethanol (yield: $2.30 \mathrm{~g}$ ). The procedure for obtaining ZIF67 was similar to that for ZIF-8, except that $\mathrm{Co}(\mathrm{OAc})_{2} \cdot 4 \mathrm{H}_{2} \mathrm{O}$ was used instead of $\mathrm{Zn}(\mathrm{OAc})_{2} \cdot 2 \mathrm{H}_{2} \mathrm{O}$ (yield: $2.20 \mathrm{~g}$ ). Before adsorption, the products were dried overnight under vacuum at room temperature.

Adsorption and desorption tests: Batch adsorption experiments were performed on the prepared ZIFs adsorbent. For this, $50 \mathrm{mg}$ adsorbent was put into $50 \mathrm{~mL}$ of dye solution with various predetermined initial dye concentrations (10-110 $\mathrm{mg} \mathrm{L}^{-1}$ for $\mathrm{AB} 40,1-10 \mathrm{mg} \mathrm{L}^{-1}$ for $\mathrm{MB}$ ) in a $100 \mathrm{~mL}$ conical flasks, which were stirred at $200 \mathrm{rpm}$ on a magnetic stirrer and maintained for a fixed time $(0.5 \mathrm{~h}$ to $12 \mathrm{~h})$ at a temperature of $25 \pm 1{ }^{\circ} \mathrm{C}$. After each adsorption process, the ZIFs samples were separated by centrifugation (4000 rpm, $3 \mathrm{~min}$ ). The dye concentrations of the solutions were then measured using a UV-visible spectrophotometer at $\lambda_{\max }$ of $619 \mathrm{~nm}$ for $\mathrm{AB} 40$ and $663 \mathrm{~nm}$ for $\mathrm{MB}$, respectively. After complete adsorption (initial AB40 concentrations: $60 \mathrm{mg} \mathrm{L}^{-1}$ for ZIF-8, $90 \mathrm{mg} \mathrm{L}^{-1}$ for ZIF-67), the used adsorbents ZIF-8 and ZIF-67 were dried at the room temperature. Then the dye-uptake adsorbent (50 $\mathrm{mg}$ ) was placed in $50 \mathrm{~mL}$ regenerated solution (such as $\mathrm{H}_{2} \mathrm{O}$, ethanol, $0.025 \mathrm{~mol} \mathrm{~L}^{-1}$ aqueous $\mathrm{NaOH}$ solution and $0.025 \mathrm{~mol}$ $\mathrm{L}^{-1}$ alcoholic solution of sodium hydroxide), stirred for $15 \mathrm{~min}$ to desorb dye molecules at $70{ }^{\circ} \mathrm{C}$.

\section{RESULTS AND DISCUSSION}

Synthesis: ZIF-8 was synthesized using a solvothermal method (with methanol as solvent) as described previously ${ }^{9,22}$. ZIF-67 was also obtained using methanol as the solvent. The relative intensity and peak positions of the powder X-ray diffraction (PXRD) pattern are in agreement with previous reports ${ }^{3}$, confirming the formation of pure crystalline ZIF-8 and ZIF67 (Fig. 1).
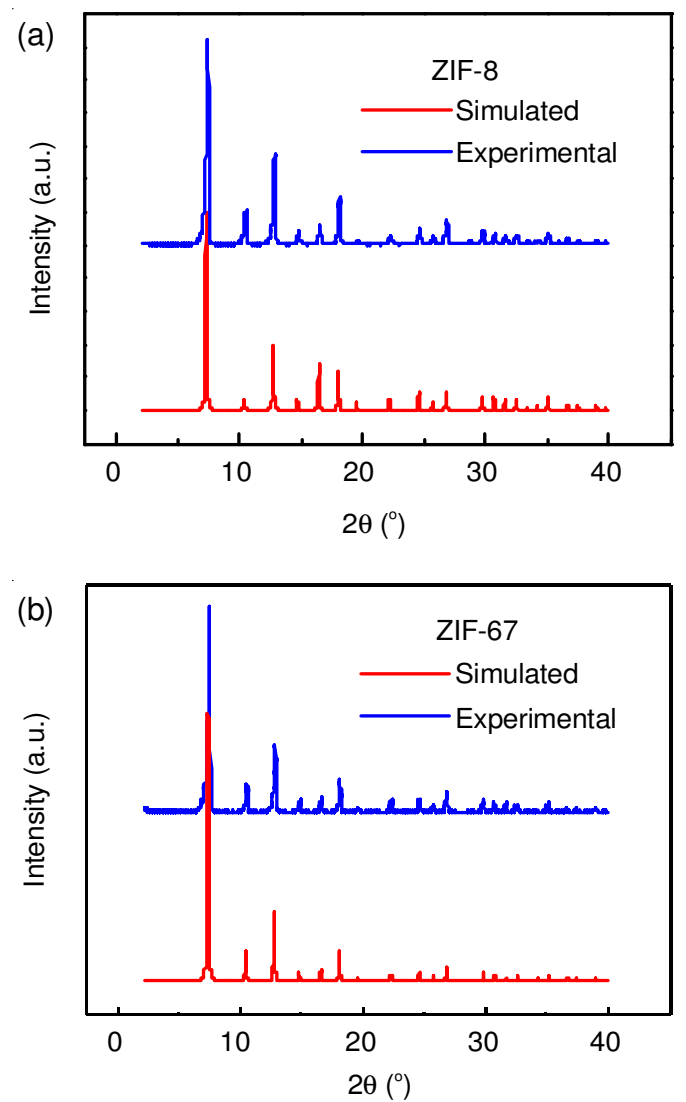

Fig. 1. Experimental XRD pattern of sample (blue pattern) and XRD pattern simulated from crystal structure data (red pattern): (a) ZIF-8, (b) ZIF-67

Adsorption properties: Fig. 2 shows the dye AB40 uptake of ZIF-8 and ZIF-67 as a function of time at different initial concentrations. The amount of AB40 adsorption increased both with increasing initial AB40 concentration and with reaction time. The results (Fig. 2) clearly indicate that the adsorption process for ZIF-8 and ZIF-67 approached the equilibrium within $6 \mathrm{~h}$ and beyond this time no significant increase in adsorption capacity was observed. This was therefore selected as the optimum contact time. The initial dye concentration had little influence on the contact time necessary to reach equilibrium. For ZIF-8, the equilibrium adsorption capacity was found to be about $22 \mathrm{mg} \mathrm{g}^{-1}$, at an initial concentration of $60 \mathrm{mg} \mathrm{L}^{-1}$ and around $55 \mathrm{mg} \mathrm{g}^{-1}$ for ZIF-67 at an initial concentration of $60 \mathrm{mg} \mathrm{L}^{-1}$.

The effect of the contact time and initial concentration on the adsorption of MB by ZIF-8 and ZIF-67 is presented in Fig. 3. It shows the contact time necessary for ZIF-8 to reach saturation to be over $2 \mathrm{~h}$; for ZIF-67 the adsorptions reach equilibrium in $c a .1 \mathrm{~h}$. The equilibrium adsorption capacity of 

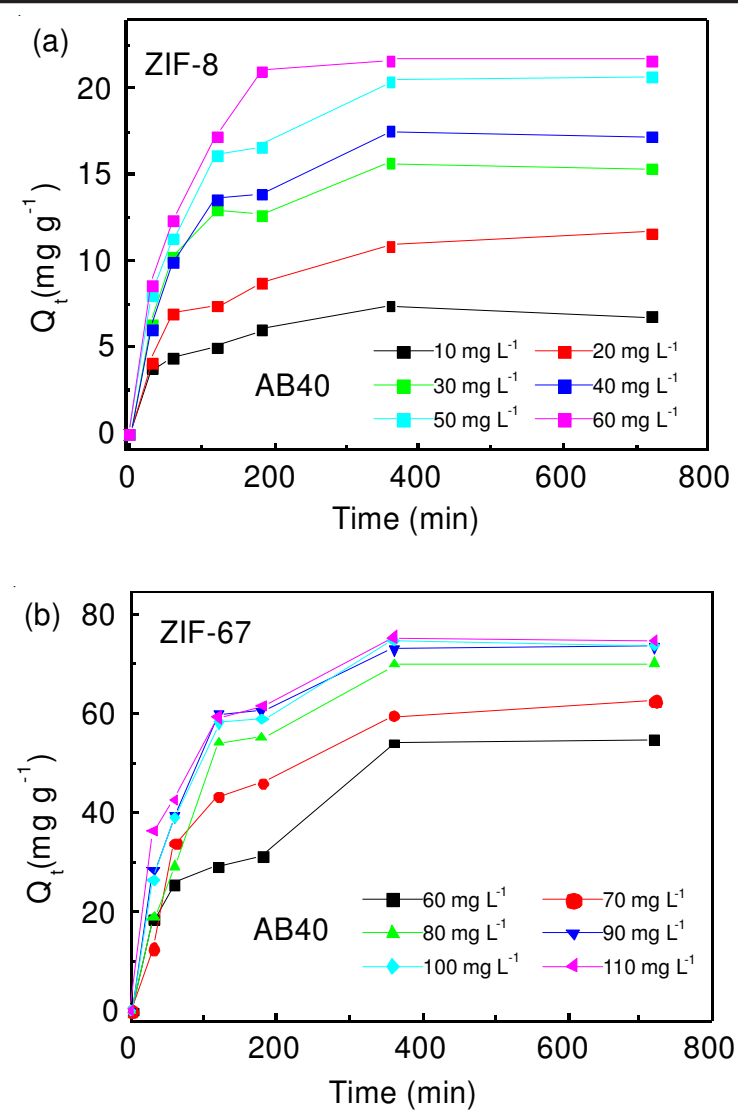

Fig. 2. Adsorption of AB40 by ZIF-8 (a) and ZIF-67 (b) as a function of time at various concentrations
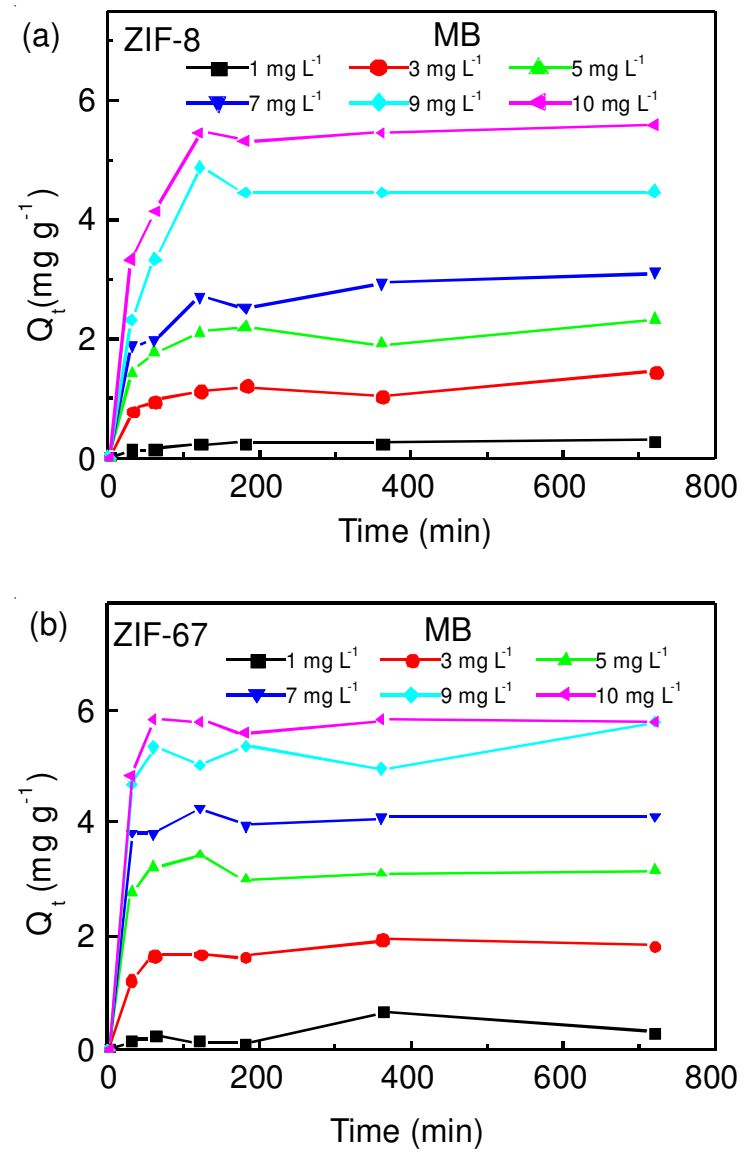

Fig. 3. Adsorption of MB by ZIF-8 (a) and ZIF-67 (b) as a function of time at various concentrations
ZIF-8 and ZIF-67 for the uptake MB was found to be about 5.4 and $5.8 \mathrm{mg} \mathrm{g}^{-1}$, respectively, at an initial concentration of $10 \mathrm{mg} \mathrm{L}^{-1}$.

The adsorption isotherms of AB40 and MB on both ZIFs were also obtained and the results are compared in Fig. 4. In fact, $\mathrm{AB} 40$ and $\mathrm{MB}$ are not readily adsorbed because it is too large to diffuse through the pore opening and fit inside the cages of the ZIF-8 and ZIF-67. It is therefore likely that the adsorption of the AB40 and MB on these ZIFs occurs initially and mainly at the external surface. Experimental samples of ZIFs are not perfect infinite lattices, but are powders of small crystallites with a significant surface area and number of defects. Chizallet et al. ${ }^{15}$ demonstrated that unsaturated $\mathrm{Zn}$ species and free $\mathrm{N}^{-}$extremities are located on the external surface of ZIF-8 or at defects. Thus, for the experimental samples ZIF-8 [Zn(mim) $\left.)_{2}\right]$ and ZIF-67 [Co(mim $\left.)_{2}\right]$ used in this study, low-coordinated zinc/cobalt cations M(II) may result in a positively charged site on the external surface of the material, while free $\mathrm{N}^{-}$moieties belonging to mim linkers may result in a negatively charged site.
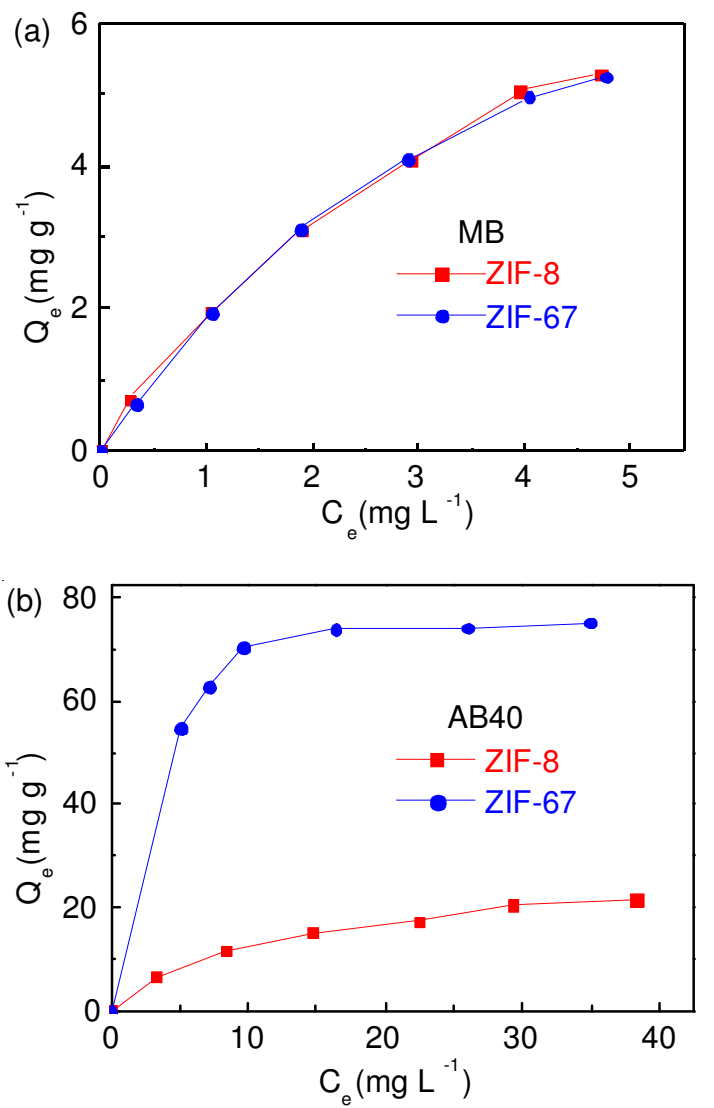

Fig. 4. Adsorption isotherms for the adsorption of MB (a) and AB40 (b) on ZIF-8 and ZIF-67

As shown in Fig. 4a, a similar adsorption capacity is observed for the uptake MB between ZIF-8 and ZIF-67. ZIF8 and ZIF-67 have the same topology and the only difference between them is the replacement of $\mathrm{Zn}$ in ZIF-8 with Co in ZIF-67. Thus, the metal ions in ZIF-8 and ZIF-67 seem to have a limited influence on the adsorption capacity for MB. In fact, $\mathrm{MB}$ is a chloride salt, it is reasonable to assume that $\mathrm{MB}$ is cationic. It is also concluded that the same $\mathrm{N}$-extremities 
(belonging to 2-methylimidazolate linkers) of ZIF-8 and ZIF67 will result in a negative charge on the external surface of the material. Thus experimental evidence indicates that electrostatic attraction is a reasonable explanation for the same adsorption capacity of MB on both ZIFs.

Contrary trend was observed for adsorption of dye AB40. As can be seen from the Fig. 4b, the uptake of anionic AB40 was different for the two ZIFs tested, with ZIF-67 exhibiting an adsorption capacity nearly three times higher than that of ZIF-8. It should be noted that the crystal morphology of ZIF8 and ZIF-67 (Fig. 5) were all aggregates composed of numerous particles ranging from 1 to $20 \mu \mathrm{m}$. In general, the surface area increased as the particle size decreased and as a consequence, the adsorption capacity increased. As crystals diameter of ZIF-67 is not less than that of ZIF-8, the large adsorption capacity over ZIF-67 cannot be explained with the sizes of the crystals. This indicates that the adsorption behaviour depends on the nature of the metal ions in ZIF-8 and ZIF-67. AB40 contains negatively charged $\mathrm{SO}_{3}{ }^{-}$groups after dissolution in water (Scheme-I). Thus, the electrostatic interaction between the framework metal cations and $\mathrm{SO}_{3}{ }^{-}$ plays a dominant role in the adsorption capacity. It seems that low-coordinated Co cations in ZIF-67 have higher affinity with the $\mathrm{SO}_{3}{ }^{-}$groups of $\mathrm{AB} 40$ molecules. In summary, the different metal ions at the external surface of ZIF-8 and ZIF-67 appear to strongly influence their adsorption capacity for AB40.
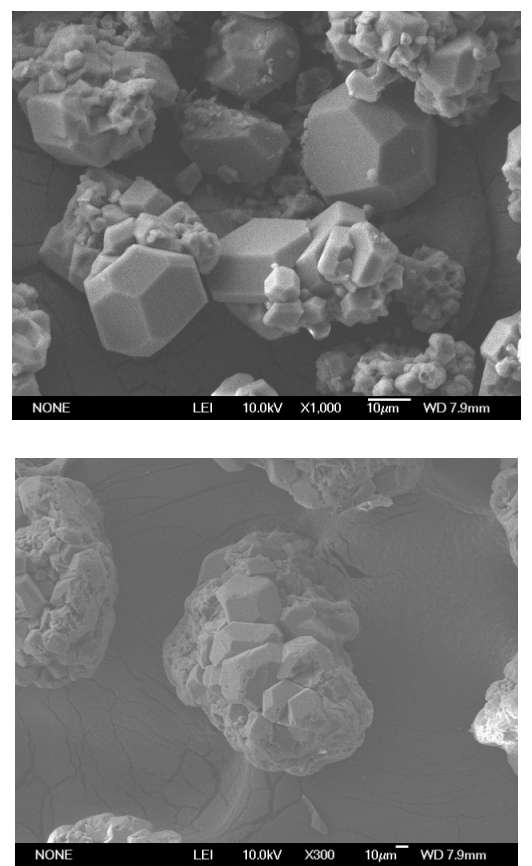

Fig. 5. SEM images of ZIF-8 (above) and ZIF-67 (below)

Desorption properties: Desorption experiments on the adsorbents with AB40 adsorbed were firstly conducted in hot aqueous solution $\left(\mathrm{H}_{2} \mathrm{O}, 70^{\circ} \mathrm{C}\right)$. As shown in Fig. 6a-b, pure water was not a good solvent for the desorption of the dye, only $13 \%$ of the AB40 was desorbed from ZIF-67 and $63 \%$ from ZIF-8, showing that only a fraction of the adsorbed AB40 can be desorbed. Organic solvents are likely to compete with the dye for the adsorption sites on the ZIFs. Furthermore, acid
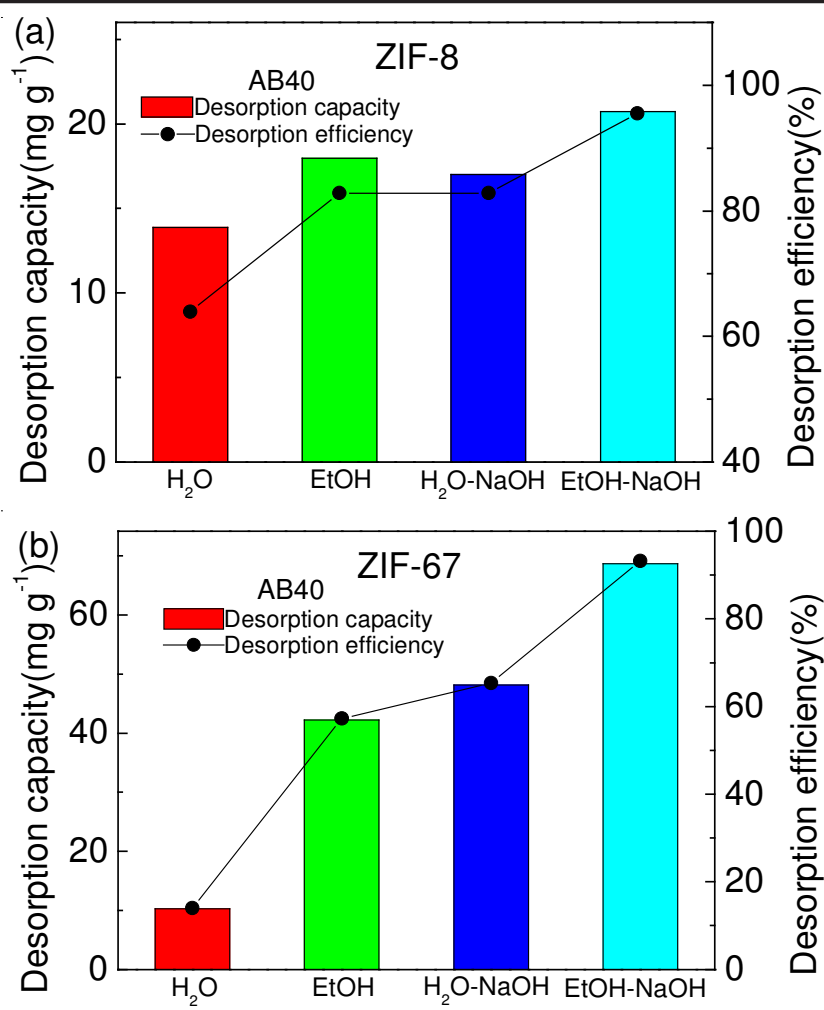

Fig. 6. Desorption capacity and desorption efficiency of AB40 on ZIF-8 (a) and ZIF-67 (b) in different medium

dyes exhibit good attraction toward basic medium ${ }^{23}$. Other medium, such as hot aqueous solution of sodium hydroxide $\left(\mathrm{H}_{2} \mathrm{O}-\mathrm{NaOH}, 70^{\circ} \mathrm{C}\right)$ and ethanol $\left(\mathrm{EtOH}, 70^{\circ} \mathrm{C}\right)$, were therefore investigated for the ability to regenerate the adsorbents. Indeed, pure ethanol or aqueous solution of sodium hydroxide was found to be more effective than pure water for desorbing AB40 from ZIF-8 and ZIF-67, as shown in Fig. 6a-b, respectively.

Higher desorption efficiency was found to be obtainable if hot alcoholic solution of sodium hydroxide (EtOH-NaOH, $70{ }^{\circ} \mathrm{C}$ ) were used as the medium. Almost complete desorption of the dye was achieved by using EtOH-NaOH medium for both ZIFs (95 and $93 \%$, for ZIF-8 and ZIF-67, respectively). This good regeneration performance can be attributed to the sufficiently competition with groups of AB40 molecules by using the EtOH-NaOH medium. The trend in desorption efficiency for the medium tested was therefore EtOH-NaOH > $\mathrm{EtOH}=\mathrm{H}_{2} \mathrm{O}-\mathrm{NaOH}>\mathrm{H}_{2} \mathrm{O}$.

\section{Conclusion}

We have described the adsorption of anionic dye acid blue 40 and cationic dye methylene blue from aqueous solution onto isotypic ZIFs such as ZIF-8 [Zn(mim) $)_{2}$ and ZIF-67 $\left[\mathrm{Co}(\mathrm{mim})_{2}\right]$. For cationic dye MB, a similar adsorption capacity is observed between ZIF-8 and ZIF-67. However, for anionic dye AB40, the adsorption capacity for ZIF-67 is nearly three times higher than that of ZIF-8. The results suggested that low-coordinated metal cations and $\mathrm{N}^{-}$anions at the external surface of ZIFs play a key role in dye adsorption and the adsorption mechanism may be explained by electrostatic interactions. The results presented here provide a perspective on the charged sites (external surface) of ZIFs by dye adsorption. Due to tunable functionality as well as exceptionally 
stability of ZIFs, this work makes significant progress towards liquid phase adsorption and separation processes by using this class of materials.

\section{ACKNOWLEDGEMENTS}

This work was financially supported by the National Natural Science Funds (Grant No. 51172153).

\section{REFERENCES}

1. Y.Q. Tian, C.X. Cai, Y. Ji, X.Z. You, S.M. Peng and G.H. Lee, Angew. Chem. Int. Ed., 41, 1384 (2002).

2. X.C. Huang, Y.Y. Lin, J.P. Zhang and X.M. Chen, Angew. Chem. Int. Ed., 45, 1557 (2006).

3. K.S. Park, Z. Ni, A.P. Côté, J.Y. Choi, R.D. Huang, F.J. Uribe-Romo, H.K. Chae, M. O'Keeffe and O.M. Yaghi, Proc. Nat. Acad. Sci. USA, 103, 10186 (2006)

4. R. Banerjee, A. Phan, B. Wang, C. Knobler, H. Furukawa, M. O'Keeffe and O.M. Yaghi, Science, 319, 939 (2008).

5. T. Wu, X.H. Bu, J. Zhang and P.Y. Feng, Chem. Mater., 20, 7377 (2008).

6. Y.L. Liu, V.C. Kravtsov, R. Larsen and M. Eddaoudi, Chem. Commun., 1488 (2006)

7. H. Wu, W. Zhou and T. Yildirim, J. Am. Chem. Soc., 131, 4995 (2009).

8. K.H. Li, D.H. Olson, J. Seidel, T.J. Emge, H.W. Gong, H.P. Zeng and J. Li, J. Am. Chem. Soc., 131, 10368 (2009).
9. H. Bux, F.Y. Liang, Y.S. Li, J. Cravillon, M. Wiebcke and J. Caro, J. Am. Chem. Soc., 131, 16000 (2009).

10. H.L. Jiang, B. Liu, T. Akita, M. Haruta, H. Sakurai and Q. Xu, J. Am. Chem. Soc., 131, 11302 (2009).

11. G. Lu and J.T. Hupp, J. Am. Chem. Soc., 132, 7832 (2010).

12. S. Basu, M. Maes, A. Cano-Odena, L. Alaerts, D.E. De Vos and I.F.J. Vankelecom, J. Membr. Sci., 344, 190 (2009).

13. J.C.S. Remi, T. Rémy, V.V. Hunskerken, S.V.D. Perre, T. Duerinck, M. Maes, D.D. Vos, E. Gobechiya, C.E.A. Kirschhock, G.V. Baron and J.F.M. Denayer, Chem. Sus. Chem., 4, 1074 (2011).

14. D.D. Ge and H.K. Lee, J. Chromatogr. A, 47, 8490 (2011).

15. C. Chizallet, S. Lazare, D. Bazer-Bachi, F. Bonnier, V. Lecocq, E. Soyer, A.-A. Quoineaud and N. Bats, J. Am. Chem. Soc., 132, 12365 (2010).

16. V.K. Gupta and Suhas, J. Environ. Manage., 90, 2313 (2009).

17. S. Ledakowicz, M. Solecka and R. Zylla, J. Biotechnol., 89, 175 (2001).

18. P.B. Moraes, R.R.L. Pelegrino and R. Bertazzoli, J. Environ. Sci. Health A, 42, 2131 (2007).

19. M. Rafatullah, O. Sulaiman, R. Hashim and A. Ahmad, J. Hazard. Mater., 177, 70 (2010).

20. M. Özacar and I.A. Sengil, Adsorption, 8, 301 (2002).

21. M.L. Fetterolf, H.V. Patel and J.M. Jenning, J. Chem. Eng. Data, 48, 831 (2003)

22. J. Cravillon, S. Münzer, Lohmeier, S.J. Feldhoff, A. Feldhoff, K. Huber and M. Wiebcke, Chem. Mater., 21, 1410 (2009).

23. A. Mittal, J. Mittal, A. Malviya and V.K. Gupta, J. Colloid Interf. Sci., 340, 16 (2009). 